\title{
Association between H19 SNP rs217727 and lung cancer risk in a Chinese population: a case control study
}

\author{
Lingling $\mathrm{Li}^{1 \dagger}$, Genyan Guo ${ }^{1 \dagger}$, Haibo Zhang ${ }^{2}$, Baosen Zhou ${ }^{3}$, Lu Bai', He Chen ${ }^{1}$, Yuxia Zhao ${ }^{1 *}$ (D) and Ying Yan²
}

\begin{abstract}
Background: H19 was the first long non-coding RNA (IncRNA) to be confirmed. Recently, studies have suggested that $\mathrm{H} 19$ may participate in lung cancer (LC) development and progression. This study assessed whether single nucleotide polymorphisms (SNPS) in $\mathrm{H} 19$ are associated with the risk of LC in a Chinese population.

Methods: A case-control study was performed, and H19 SNP rs217727 was analyzed in 555 lung cancer patients from two hospitals and 618 healthy controls to test the association between this SNP and the susceptibility to LC.

Results: The A/A homozygous genotype of rs 217727 was significantly associated with an increased LC risk (odds ratio $(\mathrm{OR})=1.661,95 \%$ confidence interval $(C I)=1.155$ to $2.388, P=0.006)$. Significant associations remained after stratification by smoking status $(P<0.001)$. Furthermore, the A/A genotype had a higher risk of LC than those of $G$ / $G$ in the squamous cell carcinoma $(O R=2.022, P=0.004)$ and adenocarcinoma $(O R=1.606, P=0.045)$ subgroups.

Conclusions: The rs217727 SNP in IncRNA H19 was significantly associated with susceptibility to LC, particularly in squamous cell carcinoma and adenocarcinoma, and identified the homozygous A/A genotype as a risk factor for LC.
\end{abstract}

Keywords: Lung cancer, H19, Susceptibility, SNP

\section{Background}

Lung cancer (LC) has a high incidence and will continue to be the most common cause of cancer-related death around the world [1]. In China, this malignancy has the highest mortality and accounts for an estimated $25 \%$ of cancerrelated deaths [2]. LC is a complex pathological process. The major risk factors by far are cigarette smoking and air pollution. Because a proportion of individuals exposed to carcinogens may have genetic factors associated with the development of cancer, predisposing genic elements should be weighed as risk factors for LC.

Long non-coding RNAs (lncRNAs) are longer than 200 nucleotides and are defined as non-protein-coding transcripts that are universally transcribed in the genome [3].

\footnotetext{
* Correspondence: zyx_yd@163.com; yanyingdoctor@sina.com 'Lingling Li and Genyan Guo contributed equally to this work. ${ }^{1}$ Department of Radiotherapy Oncology, The Fourth Affiliated Hospital of China Medical University, No.4 Chongshan East Road, Huanggu District, Shenyang, Liaoning 110032, People's Republic of China

2Department of Radiation Oncology, The General Hospital of Shenyang Military Command, No.83 Wenhua Road, Shenhe District, Shenyang, Liaoning 110016, People's Republic of China

Full list of author information is available at the end of the article
}

LncRNAs are transcribed as sense, antisense, bidirectional, intronic, or intergenic [4]. They can work by binding to chromatin-modifying complexes to specifically silence genomic loci both in cis and trans [5]. Increasingly, more studies have revealed that lncRNAs play a major role in many aspects of tumorigenesis at the epigenetic, transcriptional, and posttranscriptional levels, including cell growth, apoptosis, invasion, and metastasis. Based on the latest studies, there is evidence that lncRNAs can control gene expression through multiple mechanisms, such as transcription, translation, imprinting, genome rearrangement, and chromatin modification [6]. H19 is a maternally expressed imprinted gene on chromosome 11p15.5 that encodes for a capped and spliced RNA and has been implicated in cancer [7]. It was the first lncRNA discovered in the human genome and plays a crucial role in mammalian development $[8,9]$.

Single nucleotide polymorphisms (SNPs) have been widely used in plant, livestock, and animal genetic analyses. SNPs may affect gene expression and function. In addition, SNPs can be associated with the susceptibility to cancer. To date, there have been rare reports of 
genetic mutations in lncRNAs and their possible correlations to LC susceptibility. Thus far, the association between H19_rs217727 polymorphisms and LC has not been studied in the Chinese population.

In this hospital-based case-control study, we hypothesized a possible association between variant genotypes of the human H19 gene (rs217727) and LC. To test our hypothesis, SNPs within the H19 gene were genotyped from blood DNA samples of $555 \mathrm{LC}$ patients and 618 age- and gender-matched general population controls.

\section{Methods}

\section{Study population}

The study population consisted of $555 \mathrm{LC}$ patients and 618 healthy controls. The LC patients were consecutively recruited between September 2010 and November 2015 from the First Affiliated Hospital of China Medical University and the Fourth Affiliated Hospital of China Medical University. Each patient was histopathologically diagnosed including squamous cell carcinoma (SCC), adenocarcinoma (AD) and small cell lung cancer (SCLC). These control subjects were picked out throughout the same period in the Fourth Affiliated Hospital of China Medical University from the health examination center. Allowing for a better condition, the following exclusion criteria were used: history of LC; history of significant concomitant tumors; any cancerrelated metastasis; chemotherapy or radiotherapy; non autologous transfusion. All subjects (LC patients and healthy controls) participated had no family history of LC in this study. Then, we randomly took sample of 618 healthy controls, which were frequency matched to the LC cases on age and gender. All participants who were unrelated ethnic Chinese resided in or near Liaoning province. All individual participants voluntarily joined this study with informed consents. Information was collected by a structured questionnaire. Smoking was defined as $\geq 10$ cigarettes per day for at least 2 years.

\section{SNPs selection and genotyping}

The location of the $2.7 \mathrm{~kb}$ human $\mathrm{H} 19$ gene (Gene ID: 283120) including the DMR (differentially methylated regions) and the promoter region was pinpointed to chromosome 11, position (1972982-1981641). The HapMap project has established a common pattern in the human genome for most of the population on the basis of DNA sequence variation. Based on the HapMap data and the criteria of minor allele frequency (MAF) $>0.05$ in CB population, we found two SNPs rs217727 and rs2107425 in $\mathrm{H} 19$, and they are in high linkage disequilibrium (LD). Some researchers had found that H19_rs2107425 and H19_rs217727 play roles in carcinoma susceptibility. The role of rs2107425 polymorphism had been identified in lung cancer. So, we chose the other one SNP, rs217727.

Genomic DNA was extracted from venous blood. Usually, about $5 \mathrm{ml}$ venous blood samples were collected from each participant. The blood samples are registered and stored at $-80^{\circ} \mathrm{C}$. Genomic DNA was extracted from leukocytes, and separated from the whole blood using a standard phenol-chloroform protocol. Genotyping was performed by pre-designed TaqMan probes (Applied Biosystems, Foster City, CA, USA). The assay ID is C_ 2603707_10 (part number: 4351379), and the specific amplicon context sequence is TGTGGTGGCTGGTGGTCAACCGTCC[A/ G]CCGCAGGGGGTGGCCATGAAGATGG (Table 1) The H19_rs217727 polymorphism was amplified and genotyped through the TaqMan SNP Genotyping Assay by using the ABI 7500 Real-time PCR system (Applied Biosystems, Foster City, CA, USA) in 96-well plates. The reaction mixture $(5 \mu \mathrm{l})$ contained $2.5 \mu \mathrm{l} \mathrm{TaqMan}{ }^{\oplus}$ Genotyping Master Mix (Applied Biosystems, Foster City, CA, USA), $0.125 \mu \mathrm{l}$ hydrolysis probe, $1.375 \mu \mathrm{lddH} 2 \mathrm{O}$ and $30 \mathrm{ng}$ genomic DNA for each SNP, according to the following PCR protocol: $95^{\circ}$ $\mathrm{C}$ for $10 \mathrm{~min}$ for 1 cycle; $95^{\circ} \mathrm{C}$ for $15 \mathrm{~s}$ and $60^{\circ} \mathrm{C}$ for $1 \mathrm{~min}$ for 40 cycles; followed by a cycle of $60^{\circ} \mathrm{C}$ for $1 \mathrm{~min}$ which is a stage of analysis for genotypes. Controls (known genotype and water) were included in each reaction plate to ensure that the genotyping were accuracy. The deionized water was used as a negative control and the rs3219073/ GG SNP of PARP-1 was used as a positive control, which was previously detected in many lung cancer samples [10]. Two researchers analyses the genotype individually in a blind method. Approximately 10\% samples were randomly selected to repeat detection, the results for random sampling were $100 \%$ concordant as quality control samples.

\section{Statistical analysis}

The data obtained were computed and analyzed via SPSS, version 16.0 (SPSS Inc., Chicago, IL, USA). Continuous variables without skewness were estimated via means \pm standard derivation $(\mathrm{SD})$ and compared with the Student's $t$ tests. Categorical variables were used through frequency counts and compared by the Chi-

Table 1 Genotyped SNP ordered according to the position in the H19 gene

\begin{tabular}{lllccc}
\hline dbSNP & $\begin{array}{l}\text { NCBI assembly } \\
\text { location (Build 37) }\end{array}$ & TaqMan assay ID & Base change & Tag SNP (CHB population; HapMap) & $\begin{array}{l}\text { Cinfirmed functional } \\
\text { effect of SNP }\end{array}$ \\
\hline rs217727 & Chr.11:2016908 & C__2603707_10 & A/G & Yes & No \\
Context Sequence: TGTGGTGGCTGGTGGTCAACCGTCCIA/G]CCGCAGGGGGTGGCCATGAAGATGG & \\
\hline
\end{tabular}


square $\left(x^{2}\right)$ test [11]. The Hardy-Weinberg equilibrium (HWE) was estimated by the goodness-of- $X^{2}$ test. When the HWE was respected, the allele comparison and the additive model were asymptotically equivalent [12]. Correlations between the genotype and the susceptibility of LC were assessed via odds ratio (OR) with 95\% confidence interval (CI) by logistic regression analyses with adjustment for age and smoking status [13]. OR was also evaluated subgroup, viz. tumors of different pathological types. A value of $P<0.05$ was considered statistically significant.

\section{Results}

\section{Characteristics of the study population}

The demographics of the 555 LC patients and 618 healthy controls are summarized in Table 2. The majority of the LC patients were diagnosed with adenocarcinoma (44.6\%) followed by squamous cell carcinoma (38. $7 \%)$ and small cell carcinoma (16.7\%). The mean ages of the LC patients and healthy controls were $60.15 \pm 9.896$ and $60.05 \pm 10.170$ years, respectively. There was no significant difference in the frequency distributions of age or gender $(P=0.517$ and 0.798$)$ between the LC patients and the controls. However, the data was significantly higher $(61.8 \%)$ in cases with the smoking status than that in controls $(P<0.001)$, which is consistent with the epidemiological distribution of LC.

\section{H19 polymorphisms and the susceptibility of LC}

The genotype of H19_rs217727 and its association with the risk of LC are presented in Table 3. The genotype distribution of the rs217727 in the controls did not deviate from those expected under HWE $(P=0.167)$. A statistically significant increase in the risk of LC was found for carriers of the A/A genotype compared to the homozygous carriers of the wild-type $\mathrm{G} / \mathrm{G}$ genotype $(\mathrm{OR}=1$. $661,95 \% \mathrm{CI}=1.155-2.388, P=0.006)$. After adjustment for the smoking status, the A/A genotype was also significant $(P=0.002)$. However, when the combined A/ $\mathrm{G}+\mathrm{A} / \mathrm{A}$ genotypes were compared to the wild-type $\mathrm{G} / \mathrm{G}$ genotype, there was no significant difference.

\section{Stratified analysis of $\mathrm{H} 19$ polymorphisms and the risk of LC}

We carried out stratified analysis to assess the relationship between the H19 lncRNA SNPs and the risk of LC according to the pathological subtypes (Table 4). We found that the rs217727 A/A genotype was associated with an increased cancer risk for squamous cell carcinoma $(P=0.004$, adjusted $\mathrm{OR}=1.996,95 \% \mathrm{CI}=1.142$ to 3.489, $P=0.015)$ and adenocarcinoma $(P=0.045$, adjusted $\mathrm{OR}=1.767,95 \% \mathrm{CI}=1.096$ to $2.850, P=0.019)$, but not for small cell carcinoma $(P=0.123$, adjusted $\mathrm{OR}=1.799,95 \% \mathrm{CI}=0.846$ to $3.827, P=0.127$ ). There was no significant association between this polymorphism and the susceptibility of LC with other genotypes.

\section{Discussion}

LncRNAs, which characterize a functionally varied class of transcripts, have been found in many different species, such as humans, animals, plants, yeast, and viruses [14-17]. Many researchers suggest that lncRNAs play a key role in tumorigenesis and during cellular development, differentiation, and many other biological processes. Furthermore, several studies have reported that lncRNAs are misregulated in various types of cancers [18-20]. Significant overexpression of IncRNAs-CCAT2 was found in lung adenocarcinoma [21]. Nie et al. [22] reported that the lncRNA ANRIL was overexpressed in NSCLC patient tissues and associated with advanced "tumor node metastasis (TNM)" subsets, tumor size, and prognosis. Therefore, abnormalities of the expression of IncRNAs may be involved in the tumorigenesis of LC. Genetic variants in lncRNAs could be a biomarker for the prediction of cancer susceptibility in humans. Liu et al. [23] found that lncRNAs-MALAT1_rs619586

Table 2 Baseline characteristics of study subjects

\begin{tabular}{llll}
\hline Characteristics & Case $(n=555)$ & Controls $(n=618)$ & \\
\hline Age (year) & $60.15 \pm 9.896$ & & \\
Sex & & & \\
Male (\%) & $394(70.1)$ & $434(70.2)$ \\
$\quad$ Female (\%) & $161(29.9)$ & $184(29.8)$ \\
Smoking status & & \\
$\quad$ Ever (\%) & $343(61.8)$ & $143(23.1)$ \\
$\quad$ Never (\%) & $212(38.2)$ & $475(76.9)$ \\
Pathological types & & \\
$\quad$ Squamous Cells Carcinoma (\%) & $215(38.7)$ & - \\
Adenocarcinoma (\%) & $248(44.6)$ & - \\
Small Cell Carcinoma (\%) & $92(16.7)$ & - \\
\hline
\end{tabular}


Table $3 \mathrm{H} 19$ polymorphisms (rs217727) among the cases and controls and the associations with risk of LC

\begin{tabular}{|c|c|c|c|c|c|c|}
\hline Genotype & Case, $n(\%)$ & Control, $n(\%)$ & Crude OR, $(95 \% \mathrm{Cl})$ & $P$ value & Adjusted OR, $(95 \% \mathrm{Cl})^{\mathrm{a}}$ & $P^{a}$ value \\
\hline $\mathrm{G} / \mathrm{G}$ & $210(37.9)$ & $246(39.8)$ & 1.0 (ref.) & & 1.0 (ref.) & \\
\hline$A / G$ & $250(45.0)$ & $305(49.4)$ & $0.960(0.749 \sim 1.231)$ & 0.749 & $0.957(0.730 \sim 1.255)$ & 0.751 \\
\hline $\mathrm{A} / \mathrm{A}$ & $95(17.1)$ & $67(10.8)$ & $1.661(1.155 \sim 2.388)$ & 0.006 & $1.849(1.248 \sim 2.740)$ & 0.002 \\
\hline$A / G+A / A$ & $345(62.1)$ & $372(60.2)$ & $1.086(0.859 \sim 1.375)$ & 0.490 & $1.111(0.860 \sim 1.435)$ & 0.420 \\
\hline
\end{tabular}

The bold values mean statistically significance with $P<0.05$

$L C$ lung cancer, $O R$ odds ratio, $\mathrm{Cl}$ confidence interval

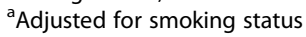

was associated with decreased hepatocellular carcinoma risk. LncRNAs-HOTAIR_rs12826786 in strong linkage disequilibrium with $\mathrm{rs} 1899663\left(\mathrm{r}^{2}=1\right)$ was associated with the risk of gastric cardia adenocarcinoma [24]. However, their definitive roles in cancer development and progression remain largely unclear.

The H19 IncRNA gene does not encode a protein, but an oncofetal RNA [25, 26]. Deregulation of oncofetal RNA plays a critical role in tumorigenesis [26]. Accumulating evidence suggests that loss of imprinting and deregulation of the H19 gene are associated with human cancer, and its overexpression is a frequent event in lung cancer development [27, 28]. H19 is abnormally expressed in many types of cancers, including gastric [29], liver [30], colorectal [31], bladder [32], and pancreatic cancer [33], and increases the tumorigenic properties of tumor cells [34-37]. In addition, studies have shown that H19 enhances invasion and migration of pancreatic ductal adenocarcinoma cells by decreasing let-7 and subsequently increasing the HMGA2-mediated epithelial-mesenchymal transition (EMT) [33]. Barsyte-
Lovejoy et al. [34] found that the knockdown of H19 inhibited colony formation and anchorage-independent growth in lung cancer cells. Other studies have reported that $\mathrm{H} 19$ could be induced under hypoxic stress through the p53/HIF1- $\alpha$ pathway. Moreover, the knockdown of H19 could significantly suppress hypoxia-induced cancer cell proliferation in vivo [36]. Furthermore, high expression of H19 was positively associated with advanced TNM stage and was a predictor of overall survival (OS) in gastric cancer patients [38, 39]. Studies have shown that the H19_rs2107425 SNP was related to the susceptibility of bladder cancer, and showed a significant correlation with LC susceptibility $(P=0.02$, age under 50 years) [40, 41]. However, Riaz et al. [42] found that H19_rs2107425 did not alter H19 mRNA expression in breast cancer. Yang et al. [43] reported that the variant H19 genotypes (CT + TT rs217727, CT + TT rs2839698) were correlated with an increased risk of gastric cancer $(P=0.040, P=0.033)$, and the CT and TT genotypes in rs2839698 were also related to higher H19 mRNA levels in serum. In contrast, the rs 217727 polymorphism did

Table 4 Stratification analyses of H19 polymorphisms (rs217727) and risk of LC

\begin{tabular}{|c|c|c|c|c|c|c|}
\hline Pathological types & Case, $n(\%)$ & Control, $n(\%)$ & Crude OR, $(95 \% \mathrm{Cl})$ & $P$ value & Adjusted OR, $(95 \% \mathrm{Cl})^{\mathrm{a}}$ & $\overline{P^{a} \text { value }}$ \\
\hline \multicolumn{7}{|c|}{ Squamous Cells Carcinoma } \\
\hline $\mathrm{G} / \mathrm{G}$ & $81(37.7)$ & $217(41.6)$ & 1.0 (ref.) & & 1.0 (ref.) & \\
\hline$A / G$ & $94(43.7)$ & $252(48.3)$ & $0.999(0.705 \sim 1.416)$ & 0.997 & $0.790(0.532 \sim 1.174)$ & 0.244 \\
\hline$A / A$ & $40(18.6)$ & $53(10.1)$ & $2.022(1.247 \sim 3.279)$ & 0.004 & $1.996(1.142 \sim 3.489)$ & 0.015 \\
\hline$A / G+A / A$ & $134(62.3)$ & $305(58.4)$ & $1.177(0.849 \sim 1.631)$ & 0.327 & $0.977(0.675 \sim 1.414)$ & 0.903 \\
\hline \multicolumn{7}{|l|}{ Adenocarcinoma } \\
\hline $\mathrm{G} / \mathrm{G}$ & $97(39.1)$ & $228(40.1)$ & 1.0 (ref.) & & 1.0 (ref.) & \\
\hline$A / G$ & $110(44.4)$ & $281(49.4)$ & $0.920(0.665 \sim 1.272)$ & 0.615 & $0.904(0.647 \sim 1.262)$ & 0.552 \\
\hline$A / A$ & $41(16.5)$ & $60(10.5)$ & $1.606(1.011 \sim 2.551)$ & 0.045 & $1.767(1.096 \sim 2.850)$ & 0.019 \\
\hline$A / G+A / A$ & $151(60.9)$ & $341(59.9)$ & $1.041(0.767 \sim 1.412)$ & 0.797 & $1.047(0.765 \sim 1.433)$ & 0.776 \\
\hline \multicolumn{7}{|c|}{ Small Cell Carcinoma } \\
\hline $\mathrm{G} / \mathrm{G}$ & $32(34.8)$ & $187(41.6)$ & 1.0 (ref.) & & 1.0 (ref.) & \\
\hline$A / G$ & $46(50.0)$ & $216(48)$ & $1.245(0.761 \sim 2.035)$ & 0.383 & $1.114(0.680 \sim 1.926)$ & 0.612 \\
\hline $\mathrm{A} / \mathrm{A}$ & $14(15.2)$ & $47(10.4)$ & 1.741 (0.860 3.522) & 0.123 & $1.799(0.846 \sim 3.827)$ & 0.127 \\
\hline$A / G+A / A$ & $60(65.2)$ & $263(58.4)$ & $1.333(0.835 \sim 2.129)$ & 0.229 & $1.253(0.764 \sim 2.056)$ & 0.372 \\
\hline
\end{tabular}

The bold values indicate statistical significance $(P<0.05)$

$L C$ lung cancer, $O R$ odds ratio, $C l$ confidence interval

${ }^{a}$ Adjusted for smoking status 
not affect the H19 mRNA level. To the best of our knowledge, the role of the H19_rs217727 polymorphism in LC susceptibility is still unknown in the Chinese population. Accordingly, we investigated whether this polymorphism was associated with the risk of LC in the Chinese population.

In this study, the A/A genotype of H19_rs217727 was significantly higher in the LC patients than in the controls $(P=0.006)$. In particular, there was a significantly increased risk of squamous cells carcinoma $(P=0.004)$ and adenocarcinoma $(P=0.045)$. However, when the combined $\mathrm{A} / \mathrm{G}+\mathrm{A} / \mathrm{A}$ genotypes were compared with the wild-type G/G genotype, there was no significant difference. Therefore, the $\mathrm{G}$ allele may be a protective factor and people who carry this allele may be less likely to develop lung cancer. However, the present research was limited with respect to geographical variation, nation, and sample size. These factors may greatly affect the accuracy of this experiment. Additional studies that encompass more geographical regions, additional ethnic groups, and larger sample size should be performed. Although all subjects were enrolled from only two hospitals and selection bias could not be avoided, the genotype distribution of the controls in our study did accord with the HWE. Additional studies are also necessary to understand the mechanism by which the rs217727 SNP affects H19 mRNA expression, alters the translational efficiency, or leads to alterations in the H19 structure in LC.

\section{Conclusions}

In the current study, we found that the H19_rs217727 polymorphism plays a crucial role in the risk of LC in a Chinese population. Larger population-based studies are required to confirm the relationship between H19 expression levels and the susceptibility to LC. H19_ rs217727 SNPs may be potential clinical markers for predicting the risk of LC.

\section{Abbreviations}

AD: Adenocarcinoma; Cl: Confidence interval; DMR: Differentially methylated regions; HWE: Hardy-Weinberg equilibrium; LC: Lung cancer; LD: Linkage disequilibrium; IncRNA: Long non-coding RNA; MAF: Minor allele frequency; OR: Odds ratio; SCC: Squamous cell carcinoma; SCLC: Small cell lung cancer SD: Standard derivation; SNPs: Single nucleotide polymorphisms

\section{Acknowledgments}

The authors thank all patients and control subjects for their participation and all personnel participating in our study for help in preparing blood samples, interviewing subjects, analyzing data and supporting experiments.

\section{Funding}

This work was supported by the Clinical Capability Construction Project for Liaoning Provincial Hospitals (grant number LNCCC-B08-2014).

\section{Availability of data and materials}

All data generated or analyzed during this study are included in this manuscript.

\section{Authors' contributions}

$\mathrm{LL}$ analyzed and interpreted the patient data regarding the lung cancer and was a major contributor in writing the manuscript. GG screened out the gene loci and took part in writing the manuscript. $\mathrm{LB}, \mathrm{HC}$ and $\mathrm{HZ}$ participated in sample collection. BZ provided experimental conditions. BZ, YY and YZ guided the experiment. All authors read and approved the final manuscript.

\section{Ethics approval and consent to participate}

All individual participants voluntarily joined this study and provided written informed consents. Ethical approval for this investigation was obtained from the Ethics Committee of the China Medical University. Issue date: March 8, 2010.

\section{Consent for publication}

Not applicable.

\section{Competing interests}

The authors declare that they have no competing interests.

\section{Publisher's Note}

Springer Nature remains neutral with regard to jurisdictional claims in published maps and institutional affiliations.

\section{Author details}

${ }^{1}$ Department of Radiotherapy Oncology, The Fourth Affiliated Hospital of China Medical University, No.4 Chongshan East Road, Huanggu District, Shenyang, Liaoning 110032, People's Republic of China. ${ }^{2}$ Department of Radiation Oncology, The General Hospital of Shenyang Military Command, No.83 Wenhua Road, Shenhe District, Shenyang, Liaoning 110016, People's Republic of China. ${ }^{3}$ Department of Epidemiology, China Medical University, Shenyang, Liaoning, China. ${ }^{4}$ Department of Radiotherapy Oncology, The First Affiliated Hospital of China Medical University, Shenyang, Liaoning, China.

Received: 21 June 2017 Accepted: 23 March 2018

Published online: 02 August 2018

References

1. Siegel R, Ma J, Zou Z, et al. Cancer statistics. CA Cancer J Clin. 2014;64:9-29.

2. Chen $\mathrm{W}$, Zheng $\mathrm{R}$, Zeng $\mathrm{H}$, et al. Annual report on status of cancer in China, 2011. Chin J Cancer Res. 2015;27:2.

3. Ponting CP, Oliver PL, Reik W. Evolution and functions of long noncoding RNAs. Cell. 2009;136:629-41.

4. Nakaqawa S, Kageyama Y. Nuclear IncRNAs as epigenetic regulators-beyond skepticism. Biochim Biophys Acta. 2014;1839(3):215-22.

5. Koziol MJ, Rinn JL. RNA traffic control of chromatin complexes. Curr Opin Genet Dev. 2010:20:142-8.

6. Fatica A, Bozzoni I. Long non-coding RNAs: new players in cell differentiation and development. Nat Rev Genet. 2014:15:7-21.

7. Kallen AN, Zhou XB, Xu J, et al. The imprinted H19 IncRNA antagonizes let-7 microRNAs. Mol Cell. 2013:52:101-12.

8. Brannan $\mathrm{Cl}$, Dees $\mathrm{EC}$, Ingram RS, et al. The product of the $\mathrm{H} 19$ gene may function as an RNA. Mol Cell Biol. 1990;10(1):28-36.

9. Keniry A, Oxley D, Monnier $\mathrm{P}$, et al. The H19 lincRNA is a developmental reservoir of miR-675 that suppresses growth and lgf1r. Nat Cell Biol. 2012;14:659-65.

10. Wang HT, Gao Y, Zhao YX, et al. PARP-1 rs3219073 polymorphism may contribute to susceptibility to lung cancer. Genet Test Mol Biomarkers. 2014;18(11):736-40.

11. Adamec C. Example of the use of the nonparametric test. Test $x^{2}$ for comparison of 2 independent examples. Cesk Zdrav. 1964;12:613-9.

12. Guedj M, Nuel G, Prum B. A note on allelic tests in case-control association studies. Ann Hum Genet. 2008;72:407-9.

13. Woolf B. On estimating the relation between blood group and disease. Ann Hum Genet. 1955;19:251-3.

14. Pauli A, Valen E, Lin MF, et al. Systematic identification of long noncoding RNAs expressed during zebrafish embryogenesis. Genome Res. 2012;22(3):577-91.

15. Swiezewski S, Liu F, Magusin A, et al. Cold-induced silencing by long antisense transcripts of an Arabidopsis Polycomb target. Nature. 2009; 462(7274):799-802.

16. Houseley J, Rubbi L, Grunstein M, et al. A ncRNA modulates histone modification and mRNA induction in the yeast GAL gene cluster. Mol Cell. 2008;32(5):685-95 
17. Reeves MB, Davies AA, McSharry BP, et al. Complex I binding by a virally encoded RNA regulates mitochondria-induced cell death. Science. 2007;316(5829):1345-8.

18. Cesana M, Cacchiarelli $D$, Legnini I, et al. A long noncoding RNA controls muscle differentiation by functioning as a competing endogenous RNA. Cell. 2011;147:358-69.

19. Guffanti A, lacono M, Pelucchi $P$, et al. A transcriptional sketch of a primary human breast cancer by 454 deep sequencing. BMC. Genomics. 2009;10:163.

20. Khaitan D, Dinger ME, Mazar J, et al. Themelanoma-upregulated long noncoding RNASPRY4-IT1 modulates apoptosis and invasion. Cancer Res. 2011;71:3852-62.

21. Qiu M, Xu Y, Yang $X$, et al. Ccat2 is a lung adenocarcinoma-specific long non-coding RNA and promotes invasion of non-small cell lung cancer. Tumor Biol. 2014;35:5375-80.

22. Nie FQ, Sun M, Yang JS, et al. Long noncoding RNA ANRIL promotes nonsmall cell lung cancer cell proliferation and inhibits apoptosis by silencing KLF2 and P21 expression. Mol Cancer Ther. 2015;14:268-77.

23. Liu Y, Pan $S$, Liu L, et al. A genetic variant in long non-coding RNA HULC contributes to risk of HBV-related hepatocellular carcinoma in a Chinese population. PLoS One. 2012;7:e35145.

24. Guo W, Dong Z, Bai Y, et al. Associations between polymorphisms of hotair and risk of gastric cardia adenocarcinoma in a population of North China. Tumor Biol. 2015;36:2845-54.

25. Poirier F, Chan C, Timmons $\mathrm{P}$, et al. The murine $\mathrm{H} 19$ gene is activated during embryonic stem cell differentiation in vitro and at the time of implantation in the developing embryo. Development. 1991;113:1105-14.

26. Ariel L, Ayesh S, Periman EJ, et al. The product of the imprinted $\mathrm{H} 19$ gene is an oncofetal RNA. Mol Pathol. 1997:50(1):33-4.

27. Hibi K, Nakamura H, Hirai A, et al. Loss of H19 imprinting in esophageal cancer. Cancer Res. 1996:56:480-2.

28. Kondo M, Suzuki H, Ueda R, et al. Frequent loss of imprinting of the h19 gene is often associated with its overexpression in human lung cancers. Oncogene. 1995;10:1193-8.

29. Wang J, Song YX, Wang ZN. Non-coding RNAs in gastric cancer. Gene. 2015;560:1-8.

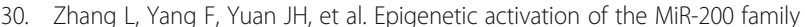
contributes to H19-mediated metastasis suppression in hepatocellular carcinoma. Carcinogenesis. 2013;34:577-86.

31. Tsang WP, Ng EK, Ng SS, et al. Oncofetal H19-derived miR-675 regulates tumor suppressor RB in human colorectal cancer. Carcinogenesis. 2010;31:350-8.

32. Luo M, Li Z, Wang W, Zeng Y, et al. Upregulated H19 contributes to bladder cancer cell proliferation by regulating ID2 expression. FEBS J. 2013;280:1709-16.

33. Ma C, Nong K, Zhu H, et al. H19 promotes pancreatic cancer metastasis by derepressing let-7's suppression on its target HMGA2-mediated EMT. Tumor Biol. 2014;35:9163-9.

34. Barsyte-Lovejoy D, Lau SK, Boutros PC, et al. The c-Myc oncogene directly induces the H19 noncoding RNA by allele-specific binding to potentiate tumorigenesis. Cancer Res. 2006;66:5330-7.

35. Lottin S, Adriaenssens E, Dupressoir T, et al. Overexpression of an ectopic $\mathrm{H} 19$ gene enhances the tumorigenic properties of breast cancer cells. Carcinogenesis. 2002;23:1885-95.

36. Ayesh S, Matouk I, Schneider T, et al. Possible physiological role of H19 RNA Mol Carcinog. 2002;35:63-74.

37. Matouk IJ, Mezan S, Mizrahi A, et al. The oncofetal H19 RNA connection: hypoxia, p53 and cancer. Biochim biophys Acta. 2010;1803:443-51.

38. Yang F, Bi J, Xue $X$, et al. Up-regulated long non-coding RNA H19 contributes to proliferation of gastric cancer cells. FEBS J. 2012;279:3159-65.

39. Zhang EB, Han L, Yin DD, et al. C-Myc-induced, long, non-coding H19 affects cell proliferation and predicts a poor prognosis in patients with gastric cancer. Med Oncol. 2014;31:914.

40. Verhaegh GW, Verkleij L, Vermeulen SH, et al. Polymorphisms in the h19 gene and the risk of bladder cancer. Eur Urol. 2008;54:1118-26.

41. Gong W-J, Yin J-Y, Li X-P, et al. Association of well-characterized lung cancer IncRNA polymorphisms with lung cancer susceptibility and platinum-based chemotherapy response. Tumor Biol. 2016;37:8349-58.

42. Riaz M, Berns EM, Sieuwerts AM, et al. Correlation of breast cancer susceptibility loci with patient characteristics, metastasis-free survival, and mRNA expression of the nearest genes. Breast Cancer Res Treat. 2012;133(3):843-51.

43. Yang C, Tang R, Ma X, et al. Tag SNPs in long non-coding RNA H19 contribute to susceptibility to gastric cancer in the Chinese Han population. Oncotarget. 2015;6(17):15311-20.

\section{Submit your next manuscript to BioMed Central and we will help you at every step:}

- We accept pre-submission inquiries

- Our selector tool helps you to find the most relevant journal

- We provide round the clock customer support

- Convenient online submission

- Thorough peer review

- Inclusion in PubMed and all major indexing services

- Maximum visibility for your research

Submit your manuscript at www.biomedcentral.com/submit 